\title{
Riding the Technology Wave: Effective Dashboard Data Visualization
}

\author{
Lisa Pappas and Lisa Whitman \\ SAS Institute, Inc., 701 SAS Campus Drive, T31, Cary, NC 27513, USA \\ \{Lisa.Pappas, Lisa.Whitman\} @sas.com
}

\begin{abstract}
Riding the technology wave, we are awash in data. Attempts to stem the tide, or at least to manage its flow, have led to a proliferation of dashboards. With data dashboards, organizations consolidate important data in a single place, typically accessed via web browser. Dashboard contents may be tables, graphics, or visual key performance indicators (KPIs). While dashboards proliferate, displaying actionable data to support decisions, they are often developed by technical professionals inexperienced in human-computer interaction design. Research abounds on visual perception, but typically this is in the context of individual entities rather than composites. This research will survey corporate and institutional dashboards and their use of particular methods of data visualization. Building on existing research into effectiveness of interactivity in diagrams, the authors seek to identify which types of data are best represented in what types of visualizations and best practices for displaying multiple visualizations in a single view.
\end{abstract}

\section{Introduction to Data Dashboards for Business Guidance}

With ever-increasing data volumes and technology accelerating the speed of commerce, business leaders struggle to distill information into actionable intelligence. To make fact-based decisions, they need the right data, delivered reliably, in an easily accessed and perceivable form. The most typical form for this is the dashboard, a metaphor adopted from automobile or aircraft designs. But this metaphor - itself based on current situational data, such as speed and miles traveled - is limited to a snapshot of separate measures.

Although current status is important, particularly for operational data, decision makers require data in context to truly manage performance over time,. They require comparisons of current values to past performance and to future objectives. Time horizon and scope of data needed differ significantly based on roles in the organization. An executive, focused on achieving enterprise-wide strategic goals, requires a high-level view across lines of business and covering months or years. Line-of-business managers, tasked with meeting tangible daily or weekly performance goals, require not only a narrower timeframe and band of data, but also, if current rates are off-target, the ability to quickly dig beneath the surface to investigate the amount and cause of variance. Business analysts have a much broader set of needs. Rather than knowing what they are looking for, they often approach performance data with as yet unformed questions. With an understanding of corporate strategy, business analysts may combine the firm's 
operational and transactional data with external data sources, such as market demographics.

In addition to time and purpose limitations, traditional data dashboards fail to leverage human perception capability to facilitate swift understanding of status and intuitive guided analysis. Factors such as placement, attention cues, cognitive load, and interactivity contribute greatly to the effectiveness of a dashboard and the ultimate value it provides to its consumers.

In this paper, we identify three categories of business data dashboards and describe their dimensions and user base. Then, we discuss elements of dashboard design, including types of visuals and interactions available, and provide guidance on their situational utility. We conclude with a discussion of further research needed, particularly driven by the rapid spread of mobile devices as a physical platform for decision support.

\section{Data Dashboard Categories}

To be a successful business tool, a dashboard must "have a direct relevance to critical business activities" [1]. It must present data efficiently and provide a means for the user to take action based on the data presented. However, critical business activities and possible actions to take vary by user role in an organization. To determine what makes dashboard visuals effective, we must understand who is using them and for what purpose. Determining the intended recipient, we can surmise the level of currency required and the types of measures or categories relevant for the role. From the purpose, we can derive the data type, quantitative or qualitative, and scope, enterprise or departmental, most likely to be presented. Among other sources, Stephen Few's Information Dashboard Design [2] provides guidance for a useful taxonomy, proposing three high level categories: strategic, operational, and analytical.

\subsection{Strategic}

The most widely recognized use of digital dashboards is that of the executive dashboard. Its purpose is to communicate to management the organization's performance relative to corporate objectives. Its nature invites comparative data, contrasting current with past performance or current to target levels.

The strategic dashboard allows for a quick overview of an organization's 'health,' so to speak; assisting with executive decisions such as the formation of long-term goals. The strategic dashboard, therefore, doesn't require real-time data: what is going on right now is not important, what is pressing is what has been going on. [3]

Strategic dashboard data may be quantitative or qualitative. Consider the concerns of an airline executive, with the dashboard shown in Figure 1. Quantitatively, the executive wants trend data on revenues, expenses, and profits. Qualitatively, s/he wants top 10 best and worst routes (based on cancellations and delays) and information that highlights successes and problem areas. 
Because of their broad time horizon yet specific targets, strategic dashboards should have an uncluttered interface, to quickly guide consumers to the answers they seek. Simply put, "Are we on track?" While such dashboards are primarily provided for senior management, they are increasingly shared to a wider audience. Providing insights on performance across the enterprise can promote alignment through lines of business toward the corporate goals.

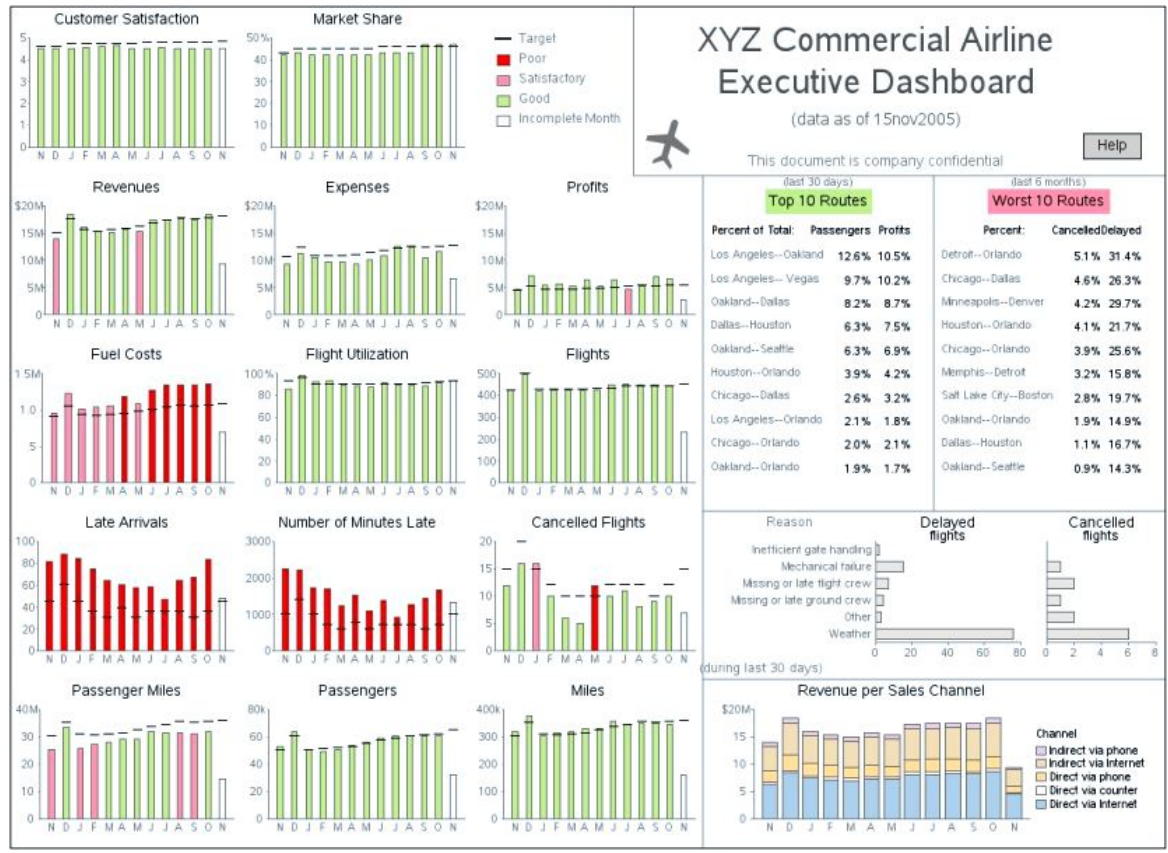

Fig. 1. Strategic Dashboard Example - Commercial Airline ${ }^{1}$

\subsection{Operational}

Monitoring operations requires more timely data, tracking constantly changing activities that could require immediate attention. Like strategic dashboards, effective operational dashboards require an uncomplicated view to enable rapid visual parsing to identify measures that are off-target and require intervention.

As with strategic dashboards, the display media on operational dashboards must be very simple. In the stressful event of an emergency that requires an immediate response, the meaning of the situation and the appropriate responses must be extremely clear and simple, or mistakes will be made [2].

Demand for currency of operational data can vary. As long as things are on track, periodic snap-shots may be sufficient. However, if a measure goes out-of-band, operational managers may want real-time data to see if the variance is an anomaly or a trend. In addition to a high level view, the operational dashboard must enable a

\footnotetext{
${ }^{1}$ Source: Used with permission of Robert Allison.
} 
deep-dive, so that the business user can examine why a measure is off-target and take appropriate action. For example, the facilities department for a university might monitor volume of water usage, as a sudden spike could suggest a leak. By drilling down on a metering graph, facilities could isolate the usage anomaly geographically.

\subsection{Analytical}

Analytical dashboards share attributes of both strategic and operational dashboards. Like the strategic dashboards, the timeframes may be wider. Like the operational dashboards, drill-down and visual exploration are essential for discovering patterns and trends in the data.

More sophisticated display media are often useful for the analyst who must examine complex data and relationships....Analytical dashboards should support interactions with the data, such as drilling down into the underlying details, to enable the exploration needed to make sense of it - that is, not just to see what is going on but to examine the causes [2].

In this regard, an analytical dashboard may exist at the intersection of strategic and operational data. For example, consider an organization conducting clinical trials for a new drug to treat diabetes. Healthcare providers at a number of sites around a metropolitan area record health statistics of trial participants at regular intervals, the operational data. But suppose that the trial sponsor, from the over-arching strategic dashboard, notices less favorable results in one particular region. The sponsor might then request a business analyst to examine the data to uncover the source of the variance. The analyst starts with regional data, but then slices the data by age, reported diet, or other variables to uncover relations between the data. For example, are participants in one region older than another, or are they more likely to smoke?

Besides looking back to examine root cause, analytical dashboards can be forward looking as well, helping to forecast outcomes. By examining current trends, business analysts can model outcomes by adjusting variables to recommend actions to optimize results.

Table 1. Summary of Dashboard Categories and Attributes

\begin{tabular}{|c|c|c|c|c|c|}
\hline Category & Purpose & $\begin{array}{l}\text { Timeframe } \\
\text { for } \\
\text { Insights }\end{array}$ & Data Scope & $\begin{array}{l}\text { Update } \\
\text { Frequency }\end{array}$ & $\begin{array}{l}\text { Interactiv } \\
\text { ity }\end{array}$ \\
\hline Strategic & $\begin{array}{l}\text { See and decide } \\
\text { or question }\end{array}$ & $\begin{array}{l}\text { Months or } \\
\text { years }\end{array}$ & $\begin{array}{l}\text { Enterprise-wide, } \\
\text { cross-business } \\
\text { unit }\end{array}$ & Moderate & Low \\
\hline Operational & See and act & $\begin{array}{l}\text { Minutes or } \\
\text { days }\end{array}$ & $\begin{array}{l}\text { Business-unit } \\
\text { specific }\end{array}$ & High & Moderate \\
\hline Analytical & $\begin{array}{l}\text { See and } \\
\text { question, } \\
\text { explore what-if } \\
\text { scenarios }\end{array}$ & $\begin{array}{l}\text { Minutes to } \\
\text { years }\end{array}$ & $\begin{array}{l}\text { Enterprise-wide, } \\
\text { cross-business } \\
\text { unit, or isolated }\end{array}$ & Low & High \\
\hline
\end{tabular}




\subsection{Summary}

Table 1 summarizes the purpose and properties of the three different categories of dashboards discussed. With an understanding of the categories of dashboards, we now explore techniques for choosing appropriate dashboard contents for the different dashboard categories and best practices for designing dashboards that are usable and effective for various types of users based on their objectives.

\section{Data Dashboard Contents and Design}

A wide variety of visuals and interactions are available for use in computer-based dashboards. In order to design a dashboard that effectively meets the needs of its audience, one must take care to choose the right visuals and interactions based on their situational utility. A dashboard is meant to be viewed at-a-glance, so once the visuals have been selected, they must be arranged in a display that can be viewed all at once, such as a computer screen, without having to scroll or navigate to multiple pages. Information is effectively integrated, risk is quickly noticed, and decisions are most easily obtained when information is displayed using visuals that are arranged together so they can be seen simultaneously on one screen. This allows for processing the information with minimal effort [4]. In the summary table above, the first purpose for each of the three categories of dashboards is to "see"; therefore, it is crucial to design dashboards so that all important information is noticed quickly.

The audience and the data should guide your design. It is important to know who will be the users of the dashboard you are designing and what their goals are, so you understand which category of dashboard you will be designing. After obtaining this information through user interviews or requirements, you can determine what story the data need to tell on the dashboard, which messages are the highest priority (i.e., data that are monitored frequently or have the most critical consequences), how the data will be presented, and what actions the users should be able to take (i.e., interactivity with the dashboard, drill-down capabilities to obtain details, hyperlinks to additional or relevant information, and communication systems like commenting). To make these decisions you will need to communicate with the users of the dashboard. They need to know what you are capable of providing so that they can understand how much data are available to them, what different ways the data can be presented and technologies that can be used, and how data can help them make decisions and accomplish their goals. They need to communicate to you what information they need to know regularly and whether they monitor particular information frequently, where they go to view data (for example, a private computer, a shared computer monitor, or a mobile device), how they will want to view the data (for example, do they prefer quickly interpreted graphics or detailed tables), and what actions they will need to take (such as, making comparisons or drilling down for details). It is also useful to understand how your audience is accustomed to viewing data and with what visuals they are already familiar in order to choose visuals on your dashboard that will be easy for them to interpret. 


\subsection{Choosing the Right Visualizations for Your Dashboard}

To design a dashboard that is effective and usable for your audience, you need to choose data visualizations that convey the message of the data clearly, are easy to interpret, avoid excessive use of space, are attractive and legible, guide the user to next steps (decide, question, or act), and allow the user to accomplish tasks to achieve their purpose (such as making comparisons or monitoring performance).

Choose visualizations that display data in a way that clearly and quickly enable users to achieve their purpose. For example, dashboards may provide the user with visualizations that compare data. Line graphs, bar charts, and bullet bars are effective visuals to use for quick comparisons. Strategic and analytical dashboards may provide interactivity, such as filtering or drill-down exploration, letting users further explore the causes of data variations noticed in the comparison. A scatter plot is another means to provide more detail behind comparisons by showing patterns created by individual data points, and bubble plots add an additional dimension in order to see what other variables might be causing variance. However, if the goal is to design an operational dashboard, then visuals showing comparisons should display any variations that would require action in a way that is quickly and easily noticeable. Making the visual a key performance indicator (KPI) is an effective way to show the comparison and also draw attention to data points that may require action. A KPI is set up to show where data falls within a specified range, so if a value falls below or above a threshold the visual utilizes color coding to draw attention to that value. Typically red is used to show when performance has fallen below a target, green indicates good performance, and yellow can be used to show that no action is required. If the audience of a dashboard is mixed or unknown, then it can be optimal to provide visuals that allow quick comparison of values while also using KPI color coding, such as the examples in Figures 2 and 3. Figure 2 combines a bar chart (which allows a user to easily compare values) with performance thresholds that reveal which categories may need action or further exploration. Figure 3 shows bullet bars, which are KPIs that allow a user to quickly compare values, alongside text that provides additional detail. If multiple KPIs are used in a dashboard, make sure that color coding is used consistently for the different KPIs, so a user does not have to go through the extra work of deciphering different color codes for KPIs that have the same meaning. For example, use the same shade of red for all KPIs on your dashboard that show if a measure is performing below a threshold [5]. The bullet bars in this example are a form of sparkline. Sparklines are visuals that, while small enough to fit alongside text, have a resolution high enough and design simple enough to help convey a message quickly [4]. Sparklines use space efficiently and can be shown next to labels or in tables. Examples of effective sparklines include bullet bars (which are effective for making comparisons when aligned in the same orientation), line charts (which, even when small, can reveal trends), and icons (such as red down arrows and green up arrows). Keeping the graphics in close proximity to relevant text helps the user to see them as related [5].

Less efficient data visualizations include pie charts, speedometers, and dials. Because these visualizations are round, they take up a lot of space relative to the amount of information that they deliver. This is an inefficient use of space when all of the visuals on a dashboard need to be on one screen. However, use of these visualizations 
may be appropriate if they are meant to draw attention to an important measure that could require immediate action or have a significant consequence. Besides being large, pie charts are also inefficient because people have difficulty comparing angles. For example, a user can scan left to right along a bar chart and compare bar heights more easily than that person could notice angle variations in pie slices. For comparisons, it is most effective to use visuals that require the user to compare line lengths with a common baseline (such as a bar chart or bullet bar) and less effective to use visuals that show comparisons with angles, area, volume or color [5]. This should be taken into consideration when choosing KPIs (such as those shown in Figure 4) for your dashboard.

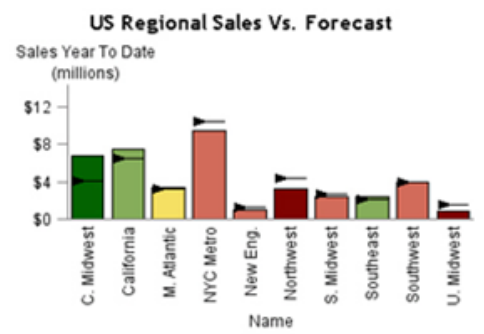

Fig. 2. A bar chart with reference lines and KPI color coding ${ }^{2}$

\begin{tabular}{|c|c|c|c|}
\hline Sports Product & ales By Coun & try & \\
\hline Product, Country & Profit $A$ & largi & \\
\hline & $0 \% 10 \% \quad 25 \%$ & $40 \%$ & \\
\hline Tennis Racket, US & -1 & & 28 \\
\hline Sleeping Bag , US & $=$ & & $7 \%$ \\
\hline Sweatshirt, GB & $=$ & & $8 \%$ \\
\hline Basketball, DE & $=$ & & $10 \%$ \\
\hline V-Neck Shirt, US & $\Longrightarrow$ & & 128 \\
\hline Roller Skates, US & $\Longrightarrow$ & & 128 \\
\hline Tennis Racket, AU & $=$ & & $16 \%$ \\
\hline Tennis Racket, AU & $=1$ & & 2096 \\
\hline Off-Court Shoes, IT & $\longrightarrow$ & & $25 \%$ \\
\hline Women's Tennis Shoes, DK & $=$ & & 328 \\
\hline Women's Glove, ES & & & $40 \%$ \\
\hline
\end{tabular}

Fig. 3. Bullet bars ${ }^{2}$

You should use your dashboard real estate as efficiently as possible. If display space is limited, use efficient visuals like bar graphs or line graphs. If a lot of space is available, you can use less efficient visuals (like dials or speedometers). Pictures should be avoided, as they do not add as much value as a data visualization, and photographs of people are distracting since people tend to fixate on any faces present on a visual display. It is best to focus on the data and avoid any visual distractions. Background images should especially be avoided, since reading text laid over them is 
difficult. Motion attracts attention, so avoid using extraneous or looping animations, such as tickers. Using too many colors, or colors that are too bright, is also distracting [6]. Since some users may be color blind, color alone should not be used to convey meaning. Instead, combine color with intensity or border thickness. Use labels to show values. If colors are used to show comparisons in data, their shades should vary. Printing your dashboard with a black and white printer is a good way to test if the colors vary enough to be distinguishable even as different shades of grey [6]. When used appropriately, color can be effective for alerting the user of any "red flags." No other colors or graphics on the dashboard should distract from alerts or color coding. Extraneous text should also be avoided. A visualization should be easy to interpret without an explanation, so only important text (like graph titles, category labels, or data values) should be on the dashboard. While a dashboard may have a small area, text should not be made so small that it is difficult to read. Ask your user to view the dashboard where it will be displayed in order to test it. This will make you aware of any adjustments that need to be made to visualizations, terminology, fonts, or color. The user should see organized groups of relevant information. Visuals that are related to each other should be close to each other, with white space around the group. The users should be able to easily find important information. Since people read left to right, put the most important information in the top-left corner, or the information that a user will want to look at first. If an object is in the center of the display, it will be noticed first, so if anything is placed directly in the center of the display, it should be important [5].
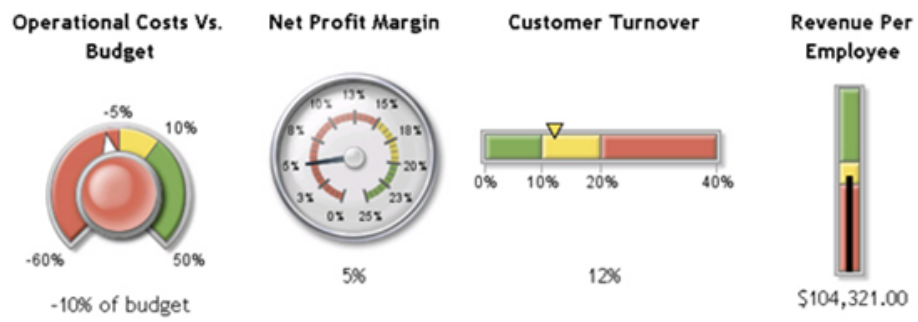

Fig. 4. Various types of KPIs - a dial, a speedometer, a slider, and a bullet bar ${ }^{2}$

Interactions can help to show the user all the information they need, even in a limited amount of space. Tooltips that appear when a user hovers the mouse pointer over a graph can provide details and even links to more information. The ability to expand or collapse visuals can be useful when many visuals crowd a small display area, allowing users to focus on the information most relevant to them. Interactions like data brushing, or interactive highlighting, can also help a user to focus their attention on important data. Data brushing is a technique where, as the user changes data selection in one view, "corresponding linked data in one or more other views is highlighted" [7]. For example, they can click on a row in a table or move a slider along a line chart (as shown in Figure 5) to reveal a bar graph showing detailed information for the date or category selected. In this way, the user can choose what is displayed on their dashboard, so that space is utilized in a way that is most beneficial to them. 


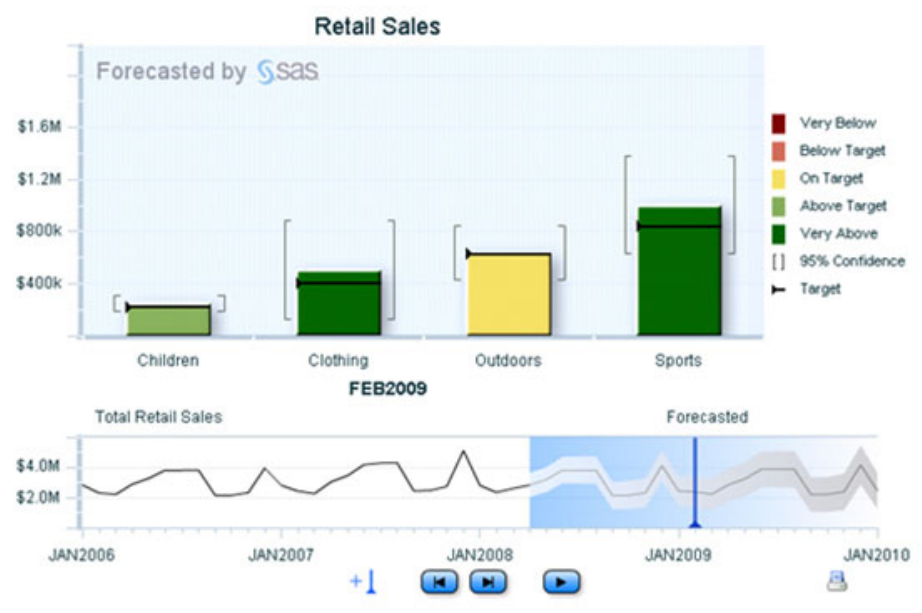

Fig. 5. An effective use of interaction for choosing information to be displayed ${ }^{2}$

\section{Conclusions and Implications for Further Research}

As described in previous sections, dashboard designers have many options when choosing methods to visualize data. But as with all effective design, they must let their design be guided by the users' goals, the context of use, and constraints - or lack thereof - of the underlying data. Availability of and reliance upon current, business critical data demands a broader skill set of designers. Rather than merely designing a visual display and specifying interactive behavior, the very task of designing is morphing and broadening. In About Face 3, Cooper, et al., propose that interface design is becoming the process of defining the product itself.

Properly deployed [design]...both identifies user requirements and defines a detailed plan for the behavior and appearance of products. In other words, design provides true product definition, based on goals of users, needs of business, and constraints of technology. [8]

The authors' supposition is particularly relevant for dashboard design, especially as business users demand access to their data on a wider variety of devices with very different form factors. The dashboard landscape now encompasses everything from mouse-driven traditional desktop monitors to smaller-screened laptops with touch pad navigation. The real estate constraints of tablet computers and smartphones are offset with gestures for direct manipulation of data visuals. Returning to our dashboard categories, we suggest:

- For an executive management strategic dashboard, use efficient visuals that combine multiple indicators in one space and provide some interactivity, such as data brushing, for limited exploration of trends and data relationships. These may be effectively included on a range of devices.

\footnotetext{
${ }^{2}$ Source of Figures 2-5: SAS BI Dashboard, http://www.sas.com/technologies/bi/entbiserver
} 
- For line-of-business managers who focus on a few truly key measures, a smartphone deployment may be feasible. The near-real-time currency of the data, persistent connection, and alert-and-notify capability can be achieved in the small mobile devices.

- The exploratory nature of analytical or forecasting dashboards requires greater real estate, although an effective dashboard could be delivered to a tablet, provided the data throughput and supporting infrastructure were sufficient. Consider a market analyst exploring stock price fluctuations timed the release of labor or pricing statistics; he is not certain where the data will take him, so providing optional views of data can be a helpful heuristic. For example, he could view a scatterplot, and noting a cluster of points, select and zoom to the cluster; then, he may want to view just the selected range in a table or bullet chart for alternative comparisons.

Designers of business interfaces face both new challenges and opportunities. Technical capability and choices are ever-expanding, as are expectations of business data consumers who want the information they need, when they need it, in an easy-toperceive format, wherever they are. However, the opportunity thoroughly to research the need, use, and context to define a product makes the challenge worthwhile. Our understanding of human perception and learning can promote data dashboards whose elegant visuals are readily used and valued.

\section{References}

1. Fuchs, G.: Dashboard Best Practices, http://www.logixml.com/resources/ whitepapers / 04_Dashboards.pdf

2. Few, S.: Information Dashboard Design: the Effective Visual Communication of Data, pp. 40-46. O'Reilly Media, Inc, Sebastopol (2006)

3. Hetherington, V.: The Dashboard Demystified, http://www. dashboardinsight.com/articles/digitaldashboards / fundamentals/the-dashboard-demystified.aspx

4. Tufte, E.R.: Beautiful Evidence. Graphics Press, Cheshire (2006)

5. Wickens, C.D., Hollands, J.G.: Engineering Psychology and Human Performance. PrenticeHall Inc., Upper Saddle River (2000)

6. Rasmussen, N., Chen, C.Y., Bansal, M.: Business Dashboards. John Wiley \& Sons, Inc., Hoboken (2009)

7. Ward, M., Grinstein, G., Keim, D.: Interactive Data Visualization: Foundations, Techniques, and Applications, pp. 320-321. A. K. Peters, Ltd., Natick (2010)

8. Cooper, A., Reimann, R., Cronin, D.: About Face 3: The Essentials of Interaction Design, pp. 17-25. John Wiley \& Sons, Indianapolis (2007) 